\title{
LOCALIZATION OF INTRA-OCULAR FOREIGN BODY BY STAINLESS STEEL SUTURES*
}

\author{
BY \\ N. KERKENEZOV \\ Lismore, N.S.W., Australia
}

Case Report

A man aged 53 was seen on January 21, 1963, with a history of having been hit in the right eye by a foreign body some 24 hours before while tapping with a hammer a metal rod which was being turned in a lathe. During the past several hours the eye had become red and irritable.

Examination.-The visual acuity in the right eye was $6 / 4$ and that in the left $6 / 6$. The right eye was moderately injected and examination revealed a very fine perforating wound of the cornea at 3 o'clock about $4 \mathrm{~mm}$. in from the limbus. The slit lamp showed but few cells in the anterior chamber. The pupil dilated freely, the lens and vitreous were both clear, and no intra-ocular foreign body was visible.

The left eye was normal on full ocular examination.

Routine $x$ rays showed the presence of an opaque minute intra-ocular foreign body in the right eye and this was then localized with a corneo-scleral ring. The foreign body could not be seen in the postero-anterior view, as its minute shadow was hidden behind that of the ring itself (Fig. 1). It was only by tilting the eye out of a true postero-anterior view that the foreign body was visible immediately adjacent to the ring (Fig. 2). No difficulty was encountered in locating the position of the foreign body in the lateral view. Localization was determined on these films and the scleral incision was made $2 \mathrm{~mm}$. behind the limbus and $3 \mathrm{~mm}$. from the mid-line where the foreign body was thought to lie in the ciliary body. After repeated unsuccessful attempts at removal with a magnet (Newton Victor P.M.X. Ophthalmic Magnet), the patient was returned to the ward for further localization.

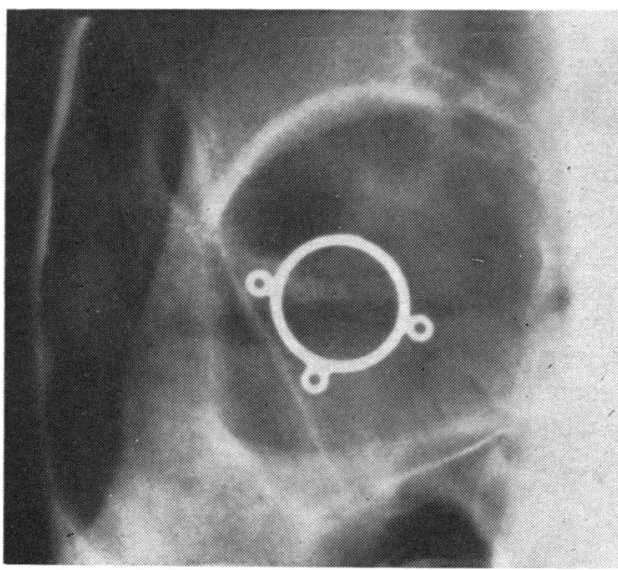

FIG. 1.-Postero-anterior $x$ ray in which the shadow of the corneo-scleral ring hides that of the minute intra-ocular foreign body.

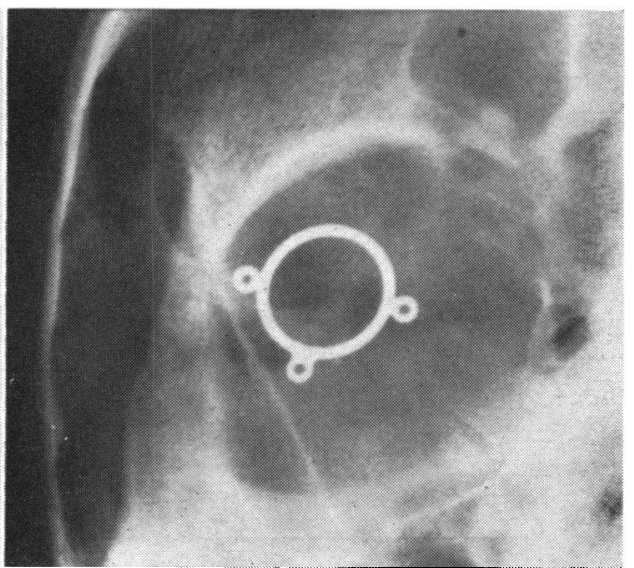

Fig. 2.- $X$ ray showing minute intra-ocular foreign body below corneo-scleral ring (medial to lower suture hole) visible only when eye is tilted out of true postero-anterior position.

12 hours later under local anaesthetic drops only, fine tantalum wire sutures were inserted at the limbus at 12, 9, and 6 o'clock, into the superficial tissues only. Knots were tied and the sutures cut short. A 6/0 wire with an eyeless needle was used for this purpose. Routine postero-anterior 
(Fig. 3) and lateral views (Fig. 4) were taken. The lateral views were facilitated by asking the patient to fix upon a point on a long rod (marked in inches) held upon the floor in contact with the $x$-ray table. The fixing eye was the normal eye. The first ray was taken while fixing upon a point thought to give a true lateral position, but in fact the three shadows of the localizing knots were not in line. The patient was then asked to fix several inches higher and the next film gave the almost true lateral picture as shown (Fig. 4). This proved an easy and quick method of obtaining this view.

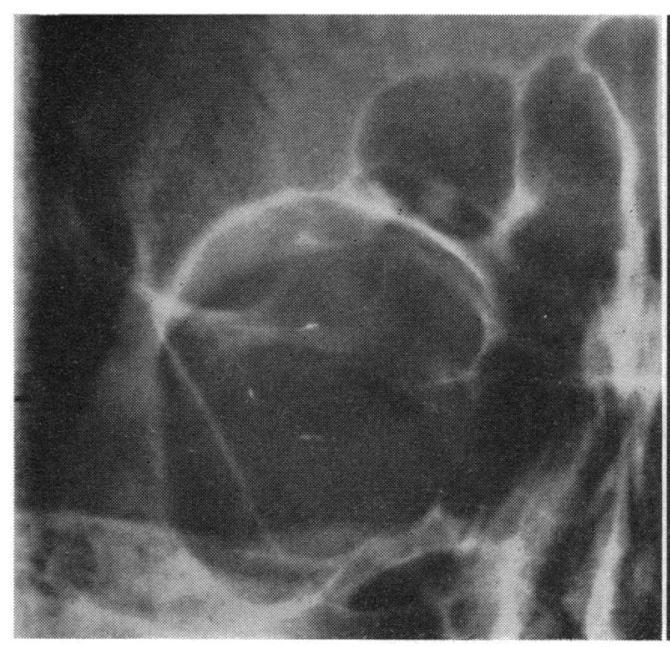

Fig. 3.-Postero-anterior $x$ ray after insertion of three tantalum wires at 12,9 , and 6 o'clock on the limbus. Adjacent and medial to the lower knot can be seen the small intra-ocular foreign body.

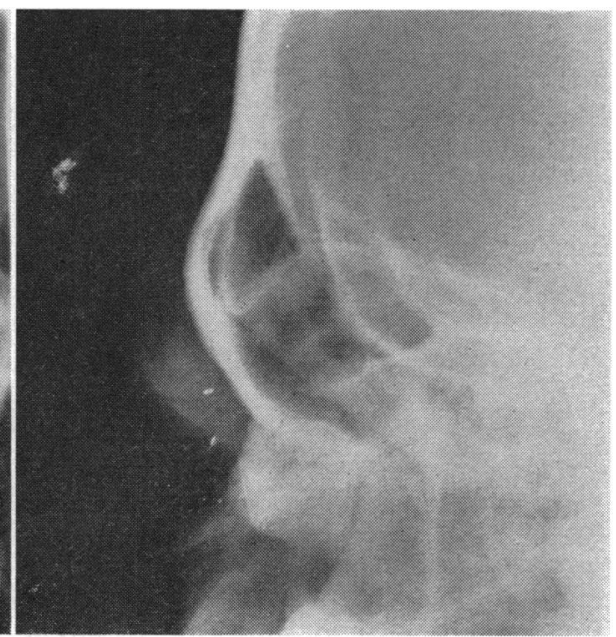

Fig. 4.-Lateral $x$ ray after insertion of tantalum wire knots at the limbus. The intraocular foreign body is to be seen almost immediately behind the shadow of the lowest knot.

Measurement by calipers of the distance between the wire knots on the eye and a corresponding measurement between their shadows on the film gave a negligible magnification factor in both postero-anterior and lateral views and this was ignored. Fig. 5 (opposite) shows a diagrammatic representation of the $x$-ray findings in which the wire knots are marked as A. 12 o'clock, B. 9 o'clock, and C. 6 o'clock. By joining these points as indicated, simple direct measurements with calipers gave the distance from $\mathrm{C}(x)$ as $1 \mathrm{~mm}$. along the limbus and the distance of the foreign body behind the limbus $(y)$ in the established meridian as $2 \mathrm{~mm}$.

In the theatre a further incision was then made in the sclera at the point indicated by the measurements above. At the time of the incision it was thought that the tip of the Graefe knife was felt to impinge upon the foreign body. The wire sutures were then removed with scissors. The leaving of the small wire knots until this stage, greatly facilitated the accurate location of the scleral incision. The first application of the magnet resulted in extraction of the minute piece of steel. A suture was placed to close the scleral incision and a small temporary conjunctival flap made to cover the scleral area and the adjacent cornea.

The patient made an uninterrupted recovery and when last seen on June 4, 1963 (approximately 5 months after the injury) the eye was white, the lens was normal, and the fundus showed no abnormality. The corrected visual acuity in the injured eye was $6 / 4$.

\section{Comment}

Very accurate localization of an intra-ocular foreign body (particularly a minute one) is of extreme importance to facilitate the removal of such a foreign body and to save the eye. The foreign body in this case was so small that even an error of $2 \mathrm{~mm}$. in localization resulted in failure to extract the foreign body at the first attempt. 


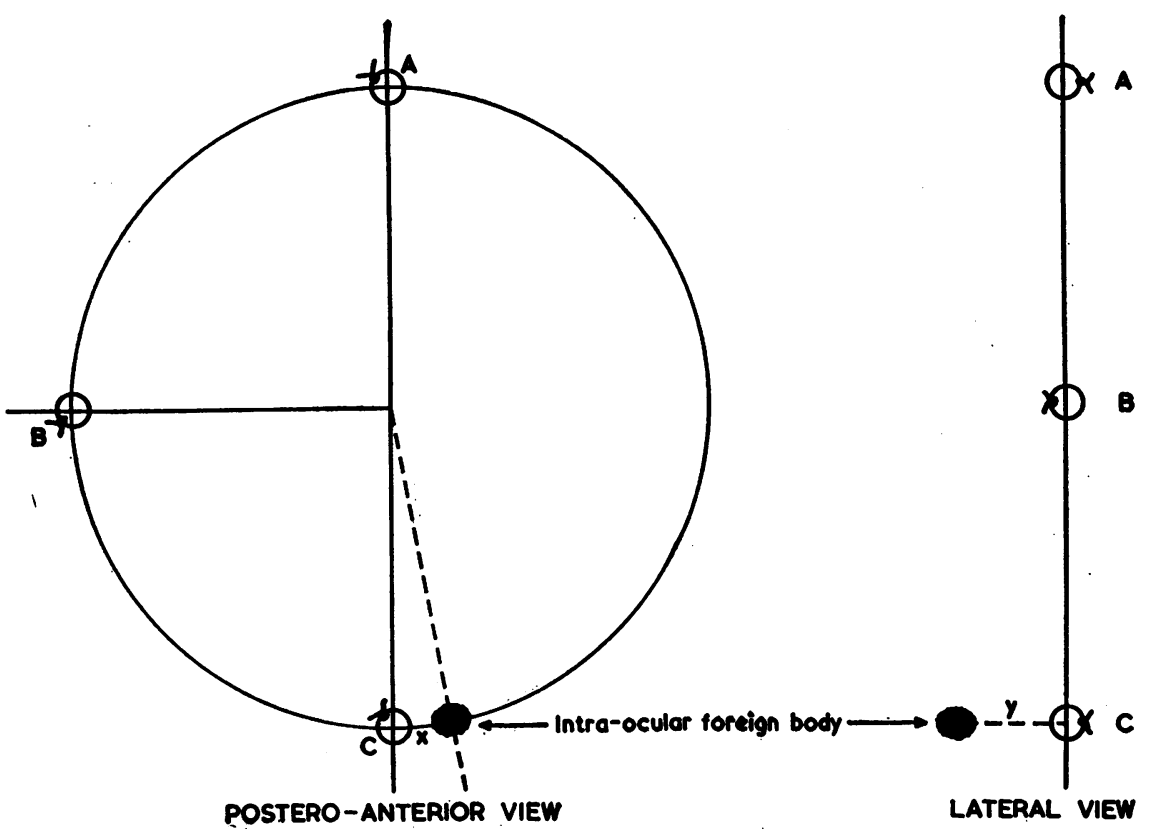

Fig. 5.-Diagram postero-anterior and lateral $x$ rays, showing localization of intra-ocular foreign body.

Of the many localization techniques, probably Sweet's method (Sweet, 1898; Reid, 1957) and the application of a corneo-scleral ring are the most commonly used in ophthalmic centres. Although in this non-industrial area the use of the corneoscleral ring in the past has proved far more accurate than Sweet's technique, it, too, has its errors and disadvantages:

(1) It is difficult always to select a ring of correct size.

(2) There is difficulty in suturing the ring accurately in position.

(3) The ring moves slightly, despite the sutures, when pressed upon by the lid margins.

(4) The bulk of the ring may hide the intra-ocular foreign body in its shadow (as in our case).

(5) The ring requires to be removed before operation, thus destroying the accurate localizing points on the eye.

(6) It requires the presence of an ophthalmic surgeon to apply the ring to the limbal area.

In Sweet's technique the localization of the intra-ocular foreign body lies solely in the hands of the radiological department without the assistance of the ophthalmologist. This rather than its accuracy would appear to be the main reason why Sweet's method has become so popular. Any localization technique which cannot repeatedly place radio-opaque localizers in direct and fixed contact with the globe cannot, however, give consistent and accurate results.

Various techniques have been employed to attach radio-opaque localizers to the globe direct, but they have all had their various disadvantages. The use of wire as in the case recorded above proved very successful indeed. With the finer, more flexible wire and improved eyeless needles now available (see below), the technique is one which $I$ feel warrants attention for the following reasons:

(1) The sutures are easy to apply (eyeless needle) with the use of local anaesthetic drops only (tetracaine hydrochloride). 
(2) The localizers can consistently be placed very accurately at the limbal area in all cases.

(3) The knots are not displaced by movement of the globe against the lids.

(4) The shadows cast in the $x$ ray by the wire are not large enough to hide an intra-ocular foreign body, even if they almost overlap as in the case reported.

(5) They remain in position until immediately before the use of a magnet, so that the position of the scleral incision can be very accurately determined (by means of calipers) from one small fixed point.

(6) They are readily removed.

(7) Localization is most accurate because of the small size of the radio-opaque localizers.

(8) The magnification factor in both the postero-anterior and lateral views is readily obtained in each case by direct measurement with calipers of the distance between the wire knots on the globe and their corresponding images on the $x$-ray film.

(9) The radiological films are very easy to interpret in contradistinction to the Sweet's technique.

Localization may be further simplified by the use of a fourth suture at 3 o'clock in addition to those used at 12 o'clock, 9 o'clock, and 6 o'clock.

The main disadvantage of this method is the need for the presence of an ophthalmologist to insert the metal sutures before the radiological localization. In most cases, however, this simple procedure can be delegated to a resident medical officer of the ophthalmic staff, and even if this is not possible the time spent by the surgeon himself is well compensated by the time saved at operation and the successful outcome for the patient.

Since the above case was recorded several other cases of intra-ocular foreign body have reached my hands and it has now been found that the ideal suture is not tantalum wire, as used in this case, but a stainless steel multistrand wire (Flexon 4/0 with an atraumatic $3 / 8$ circle needle). This is far easier to insert because of its extreme flexibility and is more readily distinguished in the $x$-ray plates. This is the suture now recommended.

\section{Summary}

A case of a minute intra-ocular foreign body is reported. Difficulty in localization was experienced with the corneo-scleral ring technique because the shadow of the intra-ocular foreign body lay behind that of the corneo-scleral ring.

Fine tantalum wire sutures (eyeless needle) were inserted at the limbus at 12 o'clock, 9 o'clock, and 6 o'clock and knots tied. These were used as radio-opaque localizers in the routine postero-anterior and lateral views.

With further experience tantalum wire has now been replaced by stainless steel multistrand 4/0 wire with an atraumatic needle (Flexon). This is the suture now recommended.

The technique is quite simple and accurate. The localizers are left in position to facilitate the planning of the scleral incision and are not removed until immediately before the application of the electromagnet.

The technique is especially recommended in the case of minute intra-ocular foreign bodies.

\section{REFERENCES}

ReID, R. G. (1957). In “Text-book of $X$-ray Diagnosis”, ed. S. C. Shanks and P. Kerley, 3rd ed., vol. 1, chap. 15, p. 395.

SweEt, W. M. (1898). Amer. J. med. Sci., 116, 320 (Cited by Reid, 1957). 